\title{
Constructive Interconnection and Damping Assignment for Port-controlled Hamiltonian Systems
}

\author{
K. Nunna, M. Sassano and A. Astolfi
}

\begin{abstract}
The Interconnection and Damping Assignment Passivity-Based Control (IDA-PBC) problem for port-controlled Hamiltonian systems is revisited. We propose a methodology that exploits the novel notion of algebraic solution of the socalled matching equation. This notion is instrumental for the construction of an energy function, defined on an extended statespace, which does not rely upon the solution of any partial differential equation. This yields, differently from the classical solution, a dynamic state feedback that stabilizes a desired equilibrium point. In addition, conditions that allow to preserve the port-controlled Hamiltonian structure in the extended closedloop system are provided. The theory is validated on two physical systems: the magnetic levitated ball and a third order food-chain system. A dynamic control law is constructed for both these systems by assigning a damping factor that cannot be assigned by the classical IDA-PBC.
\end{abstract}

\section{INTRODUCTION}

Port-controlled Hamiltonian (PCH) models, introduced as a generalization of Hamiltonian systems, characterize a class of finite dissipation systems, which include for instance - but are not limited to - electro-mechanical systems. They arise from network modeling of energy-conserving lumped-parameter physical systems with independent storage elements. According to the above framework, a dynamical system is essentially described in terms of energy which may be possessed or transformed by the system itself. This formulation appears particularly useful whenever the plant to be controlled is obtained as the interconnection of possibly simpler subsystems, the individual energies of which determine the response of the resulting complex plant. Stabilization problems for this class of dynamical systems may be tackled, for instance, by means of the so-called Passivity-based control (PBC) method [34].

Passivity-based control stems from the seminal paper [32]. This well-established approach is widely employed for the regulation of $\mathrm{PCH}$ systems, yielding effective control design techniques [25] applicable to a wide class of physical systems including mechanical systems [2], [19], [22], power

K. Nunna is with the Department of Electrical and Electronic Engineering, Imperial College London, London SW7 2AZ, UK (Emails: kameswarie.nunna09@ic.ac.uk,).

M. Sassano is with the Dipartimento di Ingegneria Civile e Ingegneria Informatica, Università di Roma "Tor Vergata", Via del Politecnico 1, 00133 Rome, Italy (Email: mario.sassano@uniroma2.it).

A. Astolfi is with the Department of Electrical and Electronic Engineering, Imperial College London, London SW7 2AZ, UK and with the Dipartimento di Ingegneria Civile e Ingegneria Informatica, Università di Roma "Tor Vergata", Via del Politecnico 1, 00133 Rome, Italy (Email: a.astolfi@ic.ac.uk). systems [11], electro-mechanical systems [27], underwater vehicles [37] and mechanical systems with nonholonomic constraints [10], [33].

A formulation of PBC known as Interconnection and Damping Assignment (IDA) has been introduced in [21]. A comprehensive discussion on IDA-PBC may be found, for instance, in [24], [33]. In this scenario, control policies should be interpreted in terms of actions aimed at shaping the energy of the controlled system to match the desired behavior. This may be achieved, for instance, by modifying, by means of feedback, the structure of the interconnection between the different subsystems and/or by adding damping to the system. A useful advantage of the method is that there is a physical interpretation of the control action as insertion of virtual springs, dampers and constraints [26]. The procedure for IDA as described in [22] and [24] involves assigning a closed-loop energy function, together with a desired interconnection and damping structure, from which a static feedback law can be developed by solving a set of partial differential equations (PDEs), usually referred to as the matching equation, in the nonlinear case, and a set of linear matrix equations, in the linear case. If the matching equation can be solved then the original control system and the target dynamical system are said to match. Similar techniques have been reported for $\mathrm{PCH}$ and Lagrangian systems in [18] and [16]. The Lagrangian counterpart of the method has been reported in [7]-[9], [12], [36]. Finally, an attempt to discuss the effect of the choice of the interconnection and the damping matrices on the closedloop performances may be found in [15].

Solution to the matching equation has received a lot of interest because it remains the stumbling block in making IDAPBC a viable design methodology. In general, the solution of the matching equation is difficult to determine. In [35] the PDEs are simplified by parameterizing the target dynamics and introducing a change of coordinates in the original system. A set of sufficient conditions on the system for the solution of the PDEs to exist is reported in [8], while [5] and [6] provide techniques to solve the PDEs. Mechanical systems with underactuation degree one are investigated in [2], where it is proved that the PDEs have solutions if the inertia matrix and the force induced by the potential energy (on the unactuated coordinates) are independent of the unactuated coordinates.

The main results of this paper are achieved by making use of a dynamic extension and introducing the notion of algebraic solution of the matching equation. A similar approach has been exploited in [28] and [29] to solve optimal control/robust 
problems for nonlinear input-affine systems, in [14] for adaptive control problems and in [30] and [31] for the analysis of some properties of nonlinear control systems. This notion is instrumental for the construction of an auxiliary energy function defined in the extended state-space. The principal contribution of the paper is then twofold. We firstly propose a methodology that permits the application of the IDA-PBC to $\mathrm{PCH}$ systems without involving the solution of any partial differential equation. The approach leads to the construction of a dynamic, in place of static, control law that allows to (asymptotically) stabilize a desired equilibrium of the closedloop extended system, without, however, preserving the $\mathrm{PCH}$ structure in the closed-loop system. Then, in the second part of the paper we discuss an alternative dynamic control law that, under additional technical conditions, allows to preserve, in the closed-loop extended system, the PCH structure corresponding to the auxiliary energy function and some interconnection and damping matrices. In addition, as a consequence of the latter result, we show that, if the matching equation does not admit a (classical) solution for a desired interconnection and damping structure, the above auxiliary energy function may be exploited to construct a (classical) solution to a matching equation corresponding to interconnection and damping that can be written as the sum of the desired matrices and continuous perturbation terms vanishing at the origin.

Dynamic extension has already been considered in the same context in [4], where it has been shown that the extension leads to equivalent matching equations for the extended system and in [23], where it has been shown that there is no advantage in considering dynamic feedback from the stabilization viewpoint. Note that in [4] the authors have provided a somewhat negative result showing that the set of solutions of the static matching equation coincides with the set of solutions of the "extended matching equations". The key difference between [4] and this paper is that the approach proposed here employs the dynamic extension to circumvent partial differential equations and search for solutions of algebraic equations instead.

Preliminary results that introduce the notion of algebraic solution of the matching equation and the construction of an energy function on an extended state-space that yields a stabilizing dynamic state-feedback for nonlinear and linear systems have been presented in [17]. This paper provides a new definition of the algebraic solution, and a dynamic feedback law that preserves the $\mathrm{PCH}$ structure and ensures (local) asymptotic stability of the equilibrium. This paper also contains the proofs of the statements in [17] and two additional numerical examples.

The rest of the paper is organized as follows. Section II introduces the problem under examination. The topic of Section III is the definition of the notion of algebraic solution of the matching equation together with some basic notation. This section also discusses the construction of the extended energy function together with its properties. Such a function is instrumental for the definition of both dynamic control laws mentioned above, described in Sections IV and V, respectively. The first main result, namely the proposed stabilizing dynamic state-feedback, is discussed in Section IV for the case of nonlinear PCH systems. Sufficient conditions that allow to preserve the $\mathrm{PCH}$ structure in the extended closed-loop system are discussed in Section V. The above results are then specialized to the case of linear systems in Section VI. The theory is validated in Section VII on two physical systems, the magnetic levitated ball, and a third order food-chain system. For the magnetic levitated ball a dynamic control law to assign a damping factor in the velocity coordinate that cannot be assigned by determining a closed-form solution of the matching equations is constructed. Finally, a dynamic statefeedback controller is designed for the food-chain system by obtaining a solution to the algebraic matching equation since the matching PDE cannot be solved. The paper is concluded by Section VIII with some final comments and suggestions for future extensions.

\section{INTERCONNECTION AND DAMPING ASSIGNMENT PASSIVITY-BASED CONTROL}

Consider the class of nonlinear port-controlled Hamiltonian systems described by equations of the form

$$
\dot{x}=(J(x)-D(x)) \nabla H(x)+g(x) u, y=g(x)^{\top} \nabla H(x),
$$

where $x(t) \in \mathbb{R}^{n}$ denotes the state of the system, $u(t) \in \mathbb{R}^{m}$ is the input and $y(t) \in \mathbb{R}^{m}$ is the output, $J: \mathbb{R}^{n} \rightarrow \mathbb{R}^{n \times n}$, $J(x)=-J(x)^{\top}$ for all $x$, is the interconnection matrix, $D: \mathbb{R}^{n} \rightarrow \mathbb{R}^{n \times n}, D(x)=D(x)^{\top}$ for all $x$, is the damping matrix and $H: \mathbb{R}^{n} \rightarrow \mathbb{R}$ is the continuously differentiable Hamiltonian function. The mapping $g: \mathbb{R}^{n} \rightarrow \mathbb{R}^{n \times m}$ and the matrix-valued functions $J$ and $D$ are continuously differentiable. Moreover the mapping $g$ is assumed to be full (column) rank for all $x \in \mathbb{R}^{n}$. Note that the system (1) is passive from the input $u$ to the output $y$, provided $H$ is positive definite and $D \geq 0$, with the Hamiltonian function $H$ as storage function.

The objective of the IDA-PBC design consists in determining a control input $u$ such that a desired equilibrium point $x^{*}$ of the closed-loop system is (asymptotically) stable and that the closed-loop system is described by the equations

$$
\dot{x}=\left(J_{d}(x)-D_{d}(x)\right) \nabla H_{d}(x), y=g(x)^{\top} \nabla H_{d}(x),
$$

where $J_{d}: \mathbb{R}^{n} \rightarrow \mathbb{R}^{n \times n}$ and $D_{d}: \mathbb{R}^{n} \rightarrow \mathbb{R}^{n \times n}, J_{d}(x)=$ $-J_{d}(x)^{\top}$ and $D_{d}(x)=D_{d}(x)^{\top} \geq 0$ for all $x$, are the desired interconnection and damping matrices and $H_{d}: \mathbb{R}^{n} \rightarrow \mathbb{R}_{+}$ is the desired energy function. The desired energy must be continuously differentiable and such that $H_{d}\left(x^{*}\right)=0$ with $x^{*}$ a strict (local) minimizer of $H_{d}$.

Let $g^{\perp}: \mathbb{R}^{n} \rightarrow \mathbb{R}^{(n-m) \times n}$ denote the full rank left annihilator of the mapping $g$, i.e. $g^{\perp}(x) g(x)=0$ for all $x \in \mathbb{R}^{n}$ and rank of $g^{\perp}$ is $n-m$. The classical solution of the IDA-PBC design problem hinges upon the solution $\mathcal{K}: \mathbb{R}^{n} \rightarrow \mathbb{R}^{n}$ of the so-called matching equation, namely the system of equations

$g(x)^{\perp}\left[(J-D) \nabla H(x)-\left(J_{d}-D_{d}\right)(\nabla H(x)+\mathcal{K}(x))\right]=0$,

where $\nabla H_{d}=\nabla H+\mathcal{K}$ denotes the gradient vector of the desired energy function $H_{d}$. Note that the mapping $\mathcal{K}$ must satisfy the condition $\partial \mathcal{K} / \partial x=(\partial \mathcal{K} / \partial x)^{\top}$, thus ensuring integrability of $\mathcal{K}$. 
In the following we suppose, without loss of generality, that the equilibrium to be stabilized is $x^{*}=0$. However, we do not require that the Hamiltonian system (1) has an equilibrium at $x=0$ for $u=0$.

\section{Algebraic Solution of Matching Equations}

The main contribution of this section is the definition of the concept of algebraic solution of the matching equation (3), which provides an alternative notion of solution of (3) without involving any partial differential equation or inequality. Such algebraic solution is then instrumental for the construction of an auxiliary energy function. The latter is then employed in Section IV to construct a stabilizing dynamic control law, which however does not preserve the PCH structure, and in Section $\mathrm{V}$ to define, under additional technical conditions, a classical solution of an auxiliary IDA-PBC problem defined on an extended-state, hence preserving the $\mathrm{PCH}$ structure of the closed-loop system.

Towards this end, assume, without loss of generality, that the energy function $H$ of the system (1) is described by the equation

$$
H(x)=d+L^{\top} x+\frac{1}{2} x^{\top} \bar{H} x+h(x),
$$

with $d \in \mathbb{R}, L \in \mathbb{R}^{n}, \bar{H}=\bar{H}^{\top} \in \mathbb{R}^{n \times n}$ and $h: \mathbb{R}^{n} \rightarrow \mathbb{R}$, such that $h(0)=0,\left.\nabla h\right|_{x=0}=0$ and $\left.\nabla^{2} h\right|_{x=0}=0$. Moreover, let $J(x)=J(0)+J_{1}(x) \triangleq J_{0}+J_{1}(x)$ and $D(x)=D(0)+$ $D_{1}(x) \triangleq D_{0}+D_{1}(x)$ with $J_{0}$ and $D_{0}$ constant matrices and $J_{1}(0)=0$ and $D_{1}(0)=0$. Similarly, let $J_{d}(x)=J_{d}(0)+$ $J_{d_{1}}(x) \triangleq J_{d_{0}}+J_{d_{1}}(x)$ and $D_{d}(x)=D_{d}(0)+D_{d_{1}}(x) \triangleq$ $D_{d_{0}}+D_{d_{1}}(x)$.

Definition 1: The equilibrium $x^{*}=0$ is assignable if $g(0)^{\perp}\left(J_{0}-D_{0}\right) L=0$.

In the following we suppose that $x^{*}=0$ is assignable. Consider now a notion of solution of the system of partial differential equations (3) as detailed in the following definition. Towards this end, let the matrix-valued function $N: \mathbb{R}^{n} \rightarrow \mathbb{R}^{n \times n}$ be such that $\nabla H(x)=N(x) x+L$, i.e. $N(x) x=\bar{H} x+\nabla h(x)$.

Definition 2: Consider system (1) and fix $J_{d}=-J_{d}^{\top}$ and $D_{d}=D_{d}^{\top} \geq 0$. A $\mathcal{C}^{1}$ matrix-valued function $P: \mathbb{R}^{n} \rightarrow \mathbb{R}^{n \times n}$, $P(x)=P(x)^{\top}$ for all $x \in \mathbb{R}^{n}$, is said to be a $\mathcal{X}$-algebraic solution of (3) if the following conditions are satisfied.

(i) The matrix $\bar{H}+P(0) \triangleq \bar{H}+\bar{P}$ is positive definite.

(ii) The condition

$$
\begin{aligned}
& g(x)^{\perp}[(J-D)(L+N(x) x) \\
& \left.\quad-\left(J_{d}-D_{d}\right)(N(x)+P(x)) x\right]=0
\end{aligned}
$$

holds for all $x \in \mathcal{X} \subseteq \mathbb{R}^{n}$, where $\mathcal{X}$ is a non-empty open set containing the origin. If $\mathcal{X}=\mathbb{R}^{n}$ then $P$ is said to be an algebraic solution of (3).

Note that the mapping $x \mapsto P(x) x$ does not need to satisfy any integrability condition, namely it may not be the gradient vector of any scalar function, hence it does not constitute a solution to the original pde (3). The structure of equation (5) is particularly appealing from the computational point of view. In fact, a solution to (5) can be given in closed-form and can be shown to satisfy the requirements in Definition 2 provided additional technical conditions hold.

Proposition 1: Consider system (1) and the function $H$ in (4) with $L=0$. Let $J_{d}=-J_{d}^{\top}, D_{d}=D_{d}^{\top} \geq 0$ be such that $J_{d}-D_{d}$ is invertible and the $\mathcal{C}^{1}$ matrix-valued function $\Lambda: \mathbb{R}^{n} \rightarrow \mathbb{R}^{m \times n}$ be such that the following hold.

(i) For all $x$ in a neighborhood $\mathcal{X}$ of the origin ${ }^{1}$

$$
\begin{gathered}
\text { skew }\left\{\left(J_{d}-D_{d}\right)^{-1}[(J-D) N(x)\right. \\
-g \Lambda(x)]\}=\operatorname{skew}\{N(x)\} .
\end{gathered}
$$

(ii) The matrix $\left(J_{d_{0}}-D_{d_{0}}\right)^{-1}\left[\left(J_{0}-D_{0}\right) \bar{H}-g(0) \Lambda(0)\right]$ is positive definite.

Then the matrix-valued function $x \mapsto P(x)$ defined as

$P(x)=\left(J_{d}-D_{d}\right)^{-1}\left[\left(J-J_{d}-D+D_{d}\right) N(x)-g(x) \Lambda(x)\right]$

is a $\mathcal{X}$-algebraic solution of (3).

Proof. To begin with, it is straightforward to note that the matrix-valued function $P$ in (7) satisfies item ( $i i)$ of Definition 2, namely solves equation (5). Then, by (6) it follows that $\left(J_{d}-D_{d}\right)^{-1}(g(x) \Lambda(x)-(J-D) N(x))-$ $(g(x) \Lambda(x)-(J-D) N(x))^{\top}\left(J_{d}-D_{d}\right)^{-\top}=N(x)-$ $N(x)^{\top}$, which implies, rearranging the terms, that the matrix $P(x)$ in (7) is symmetric for all $x \in \mathcal{X} \subseteq \mathbb{R}^{n}$. Finally, item $(i)$ of Definition 2 holds by noting that $\bar{P}+\bar{H}=$ $\left(J_{d_{0}}-D_{d_{0}}\right)^{-1}\left[\left(J_{0}-D_{0}\right) \bar{H}-g(0) \Lambda(0)\right]$ and by recalling item $(i i)$ of Proposition 1.

Note that, if the matrix-valued function $N$, which is not uniquely defined, can be given a symmetric structure for all $x \in \mathbb{R}^{n}$, then the condition in (6) reduces to requiring that $\left(J_{d}-D_{d}\right)^{-1}[(J-D) N(x)-g \Lambda(x)]=$ $[(J-D) N(x)-g \Lambda(x)]^{\top}\left(J_{d}-D_{d}\right)^{-\top}$.

Remark 1: The drift vector field in equations (1) may be replaced by a vector field $f: \mathbb{R}^{n} \rightarrow \mathbb{R}^{n}$, thus abandoning the PCH interpretation of (1). Then condition (5) yields algebraic matching equations of the form $g(x)^{\perp}\left[F(x)-\left(J_{d}-D_{d}\right) P(x)\right]=0$, where the matrix-valued function $F: \mathbb{R}^{n} \rightarrow \mathbb{R}^{n \times n}$ is such that $f(x)-f(0)=F(x) x$. Note, however, that the emphasis on the PCH form of (1) is motivated by the fact that this structure may be preserved in closed-loop, as shown in Section V.

\section{Extended desired energy function}

Exploiting the notion of algebraic solution of the matching equation (3) we construct an auxiliary energy function defined on an extended state-space which is $i$ ) (locally) positive definite around the desired equilibrium point, and $i i$ ) such that its partial derivative with respect to $x$ is described by the sum of the algebraic solution and an approximation error term that depends on the mismatch between $x$ and the state of the dynamic extension, namely

$$
H_{d}(x, \xi)=H(x)-\left(L^{\top} x+d\right)+\frac{1}{2} x^{\top} P(\xi) x+\frac{1}{2}\|x-\xi\|_{R}^{2},
$$

\footnotetext{
${ }^{1}$ The notation skew $\{A\}$ describes the skew-symmetric part of the matrix $A$, i.e. skew $\{A\}=\frac{1}{2}\left(A-A^{\top}\right)$.
} 
with $\xi \in \mathbb{R}^{n}$, where $R=R^{\top}$ is a positive definite matrix to be determined and where $\|v\|_{M}^{2}$ denotes the Euclidean norm of the vector $v \in \mathbb{R}^{n}$ weighted by the positive definite matrix $M$, namely $\|v\|_{M}^{2}=v^{\top} M v$. Note that the energy function $H_{d}: \mathbb{R}^{n} \times \mathbb{R}^{n} \rightarrow \mathbb{R}_{+}$has a strict local minimizer at $(x, \xi)=$ $(0,0)$ for any $R$. In fact, by the structure of $H$ in (4), $H_{d}$ can be written as $H_{d}(x, \xi)=\frac{1}{2}\left[x^{\top}, \xi^{\top}\right] \bar{H}_{d}\left[x^{\top}, \xi^{\top}\right]^{\top}+h_{d}(x, \xi)$, where the matrix $\bar{H}_{d}=\bar{H}_{d}^{\top} \in \mathbb{R}^{2 n \times 2 n}$ is defined as

$$
\bar{H}_{d}=\left[\begin{array}{cc}
\bar{H}+\bar{P}+R & -R \\
-R & R
\end{array}\right]
$$

and $h_{d}: \mathbb{R}^{n} \times \mathbb{R}^{n} \rightarrow \mathbb{R}$ is such that $h_{d}(0,0)=0$, $\left.\nabla h_{d}\right|_{(x, \xi)=(0,0)}=0$ and $\left.\nabla^{2} h_{d}\right|_{(x, \xi)=(0,0)}=0$. Moreover, a Schur complement argument shows that the matrix $\bar{H}_{d}$ is positive definite for any $R>0$ provided item $(i)$ of Definition 2 holds. Finally, if the algebraic solution is such that $P(x)=P(x)^{\top}>0$ and $h(x)>0$ for all $x \in \mathbb{R}^{n} \backslash\{0\}$, then the extended energy function $H_{d}$ in (8) is globally positive definite.

Remark 2: An alternative definition of algebraic solution is discussed in [17], where a continuously differentiable mapping $p: \mathbb{R}^{n} \rightarrow \mathbb{R}^{n}$ is said to be an algebraic solution if it satisfies $g(x)^{\top}\left[(J-D) \nabla H(x)-\left(J_{d}-D_{d}\right)(\nabla H(x)+p(x))\right]=0$. However, mimicking the construction in (8) and according to the notion of solution introduced in [17], the extended energy function $H_{d}$ in (8) contains the term $p(\xi) x$, which may prevent $H_{d}$ from being globally positive definite.

\section{DYNAMIC INTERCONNECTION AND DAMPING Assignment PAssivity-BASED CONTROL}

In this section it is shown that the extended desired energy function $H_{d}$ in (8) permits the construction of a dynamic control law that (asymptotically) stabilizes the desired equilibrium point $x^{*}$, provided the latter is assignable. Additional conditions that allow to preserve the $\mathrm{PCH}$ structure of the closed-loop system are discussed in Section V.

To streamline the presentation of the following result define the continuous matrix-valued function $\Phi: \mathbb{R}^{n} \times \mathbb{R}^{n} \rightarrow$ $\mathbb{R}^{n \times n}$ such that $(P(x)-P(\xi)) x=\Phi(x, \xi)(x-\xi)$ and the matrix-valued function $\Delta_{R}: \mathbb{R}^{n} \times \mathbb{R}^{n} \rightarrow \mathbb{R}^{n \times n}$ as $\Delta_{R}(x, \xi)=\Psi(x, \xi)^{\top} R^{-1}(R-\Phi(x, \xi))^{\top}$, with $\Psi(x, \xi)=$ $\frac{1}{2} \partial(P(\xi) x) / \partial \xi$. Note that, according to the above definitions, the partial derivatives of the energy function $H_{d}$ in (8) are given by

$$
\begin{aligned}
\nabla_{x} H_{d} & =\nabla H-L+P(\xi) x+R(x-\xi) \\
& =(N(x)+P(x)) x+(R-\Phi(x, \xi))(x-\xi), \\
\nabla_{\xi} H_{d} & =\Psi(x, \xi) x-R(x-\xi) .
\end{aligned}
$$

Proposition 2: Consider the PCH system (1). Let $J_{d}=-J_{d}^{\top}$ and $D_{d}=D_{d}^{\top} \geq 0$. Let $P: \mathbb{R}^{n} \rightarrow \mathbb{R}^{n \times n}$ be an algebraic solution of (3). Let $R$ be such that

$$
\begin{array}{r}
\left(N^{\top}+P\right) D_{d}(N+P)+\frac{1}{2}\left(N^{\top}+P\right)\left(J_{d}+D_{d}\right) \Delta_{R}^{\top} \\
+\frac{1}{2} \Delta_{R}\left(J_{d}+D_{d}\right)^{\top}(N+P)>0,
\end{array}
$$

for all $(x, \xi)$ in a non-empty open set $\Omega \subseteq \mathbb{R}^{n} \times \mathbb{R}^{n}$ containing the origin. Consider the dynamic control law

$$
\begin{aligned}
\dot{\xi}= & -K \nabla_{\xi} H_{d}(x, \xi), \\
u= & \left(g(x)^{\top} g(x)\right)^{-1} g(x)^{\top}[-(J-D) \nabla H \\
& \left.+\left(J_{d}-D_{d}\right)\left(\nabla_{x} H_{d}-(R-\Phi(x, \xi))(x-\xi)\right)\right]+v .
\end{aligned}
$$

Then there exists $\bar{K}=\bar{K}^{\top} \geq 0$ such that $(x, \xi)=(0,0)$ is a (locally) stable equilibrium point of the closed-loop system (1)-(11) for all $K>\bar{K}$, with $v=0$. Moreover, system (1)-(11) is passive from the input $v$ to the output $y_{d}=g(x)^{\top} \nabla_{x} H_{d}$. Finally, if the system (1)-(11) is zero-state detectable with respect to $y_{d}$ then, setting $v=-\kappa y_{d}$, the point $(x, \xi)=(0,0)$ is a (locally) asymptotically stable equilibrium of (1)-(11) for all $K>\bar{K}$ and $\kappa>0$.

Proof. Consider system (1) and the energy function $H_{d}$ in (8). Recalling that $P$ is an algebraic solution of the matching equation (3) and by the structure of the control law as in (11), for all $(x, \xi) \in \mathbb{R}^{n} \times \mathbb{R}^{n}$ the closed-loop system (1)-(11) is described by

$$
\begin{aligned}
\dot{x}= & \left(J_{d}-D_{d}\right) \nabla_{x} H_{d}-\left[\left(J_{d}-D_{d}\right)\right. \\
& (R-\Phi(x, \xi))(x-\xi)]+g(x) v, \\
\dot{\xi}= & -K \nabla_{\xi} H_{d} .
\end{aligned}
$$

The term in (12) that prevents the system from being in port-controlled Hamiltonian form stems from the mismatch between the mapping $x \mapsto P(x) x$, which may not be the gradient of any scalar function, and an actual solution of the system of partial differential equations (3). Consider now the time derivative of the energy function $H_{d}$ along the trajectories of the system (12), which can be written as

$$
\dot{H}_{d}=-\left[x^{\top}(x-\xi)^{\top}\right]\left(M+C^{\top} K C\right)\left[x^{\top}(x-\xi)^{\top}\right]^{\top}+y_{d}^{\top} v,
$$

where $C(x, \xi)=[\Psi(x, \xi)-R]$ is a $n \times 2 n$ matrix with constant rank, equal to $n$, for all $(x, \xi) \in \mathbb{R}^{n} \times \mathbb{R}^{n}$ and

$$
M(x, \xi)=\left[\begin{array}{cc}
\left(N^{\top}+P\right) D_{d}(N+P) & \Gamma_{R} \\
\Gamma_{R}^{\top} & 0
\end{array}\right],
$$

with $\Gamma_{R}=\frac{1}{2}\left(N^{\top}+P\right)\left(J_{d}+D_{d}\right)(R-\Phi)$. By employing arguments similar to those introduced in [3], the inequality (10) implies that the matrix $M(x, \xi)$ is positive definite for all $(x, \xi) \in \mathbb{R}^{n} \times \mathbb{R}^{n}$ in the intersection between $\Omega$ and the null space of the matrix $C(x, \xi)$. This result, in turn, guarantees the existence of a matrix $\bar{K}=\bar{K}^{\top} \geq 0$ such that $M(x, \xi)+C(x, \xi)^{\top} K C(x, \xi)>0$ for all $K>\bar{K}$ and all $(x, \xi) \in \Omega$. Hence $\dot{H}_{d} \leq y_{d}^{\top} v$, ensuring passivity of the system (12). Therefore, the proof is concluded by noting that the definition of the input $v$ imply that the origin is a (locally) stable equilibrium point of the closed-loop system. Asymptotic stability is proved by setting $v=-\kappa g(x)^{\top} \nabla_{x} H_{d}, \kappa>0$, and exploiting the zero-state detectability property together with LaSalle's invariance principle.

Remark 3: By the arguments employed in the proof of Proposition 2 it appears evident that the assumption of zerostate detectability of the system (1)-(11) from the output $y_{d}$ 
may be replaced by assuming that the matrix $D_{d}$ is positive definite for all $x \in \mathbb{R}^{n}$.

Exploiting ideas similar to those in the previous remark, the following result shows that the condition (10) can be (locally) satisfied provided the desired damping matrix $D_{d}$ is positive definite.

Proposition 3: Consider the PCH system (1). Let $J_{d}=-J_{d}^{\top}$ and $D_{d}=D_{d}^{\top}>0$. Let $P: \mathbb{R}^{n} \rightarrow \mathbb{R}^{n \times n}$ be an algebraic solution of (3). Then there exists a non-empty open set $\Omega \subseteq$ $\mathbb{R}^{n} \times \mathbb{R}^{n}$ containing the origin such that the condition (10) holds for all $(x, \xi) \in \Omega$ and for any $R=R^{\top}>0$, hence the (asymptotic) stability claims on system (1)-(11) discussed in Proposition 2 hold.

Proof. The claim is proved by noting that $\left.\frac{\partial P(\xi) x}{\partial \xi}\right|_{(x, \xi)=(0,0)}=0$, hence $\Delta_{R}(0,0)=0$. Then, by continuity of the functions on the left-hand side of inequality (10), it follows that (10) holds in an open neighborhood of the origin provided $D_{d}$ is positive definite.

Remark 4: Since the dynamics of the auxiliary variable $\xi$ is a design parameter, the choice $\dot{\xi}=\dot{x}, \xi(0)=x(0)$ - which clearly ensures $\xi(t)=x(t)$ for all $t \geq 0$ - seems to be appealing. In fact, this selection cancels the approximation error term in the first partial derivative in (9). A similar approach has been pursued in [1] where the extended system is expressed in the error coordinates $e=x-\xi$. However, it is interesting to note that the restriction of the energy function $H_{d}$ to the invariant submanifold $\left\{(x, \xi) \in \mathbb{R}^{n} \times \mathbb{R}^{n}: \xi=x\right\}$, namely $H_{d}(x, x)=H(x)-\left(L^{\top} x+d\right)+\frac{1}{2} x^{\top} P(x) x$ does not allow to ensure stability of the zero-equilibrium of the system (1). This is due to the additional term $\left.\nabla_{\lambda} x^{\top} P(\lambda) x\right|_{\lambda=x}$ in the partial derivative of the restricted energy function $H_{d}(x, x)$. The corresponding term in (9) is dynamically compensated for in $\dot{H}_{d}$ by $\dot{\xi}=-K \nabla_{\xi} H_{d}$.

Remark 5: Differently from the classical solution of the IDA-PBC design problem, the algebraic solution does not allow in general to preserve the port-controlled Hamiltonian structure, unless additional technical conditions are satisfied, as discussed in the following section. However, the IDA-PBC design is given in terms of the solution of a PDE, representing a serious obstruction to the applicability of the standard approach outside classes of specially structured systems.

\section{Extended Port-Controlled Hamiltonian SYSTEMS}

Proposition 2 implies that the dynamic control law (11) stabilizes a desired (assignable) equilibrium of the closed-loop system without, however, preserving the $\mathrm{PCH}$ structure. In this section we provide sufficient conditions that allow to exactly preserve the $\mathrm{PCH}$ structure in (12). In addition, we employ the above result to show that, if the matching equation (3) does not admit a (classical) solution, for a given interconnection and damping structure, but an algebraic solution can be obtained, then the extended energy function $H_{d}$ is exploited to construct a classical, hence static, solution to the partial differential equation (3) corresponding to the interconnection and damping structure which can be written as the sum of the desired matrices $J_{d}$ and $D_{d}$ and some continuous perturbation terms vanishing at the desired equilibrium.

Towards this end, let

$$
\tau(x, \xi) \triangleq \varepsilon\left\|\nabla_{x} H_{d}\right\|_{M_{x}}^{2}+\left\|\nabla_{\xi} H_{d}\right\|_{M_{\xi}}^{2},
$$

with $H_{d}$ defined in (8), where $\varepsilon>0, M_{x}=M_{x}^{\top}>0$ and $M_{\xi}=M_{\xi}^{\top}>0$ are to be determined. Moreover, define

$$
\begin{aligned}
& \Gamma_{x}(x, \xi) \triangleq \frac{\rho_{R}(x, \xi)}{\tau(x, \xi)}\left(\nabla_{x} H_{d}\right)^{\top} M_{x}, \\
& \Gamma_{\xi}(x, \xi) \triangleq \frac{\rho_{R}(x, \xi)}{\tau(x, \xi)}\left(\nabla_{\xi} H_{d}\right)^{\top} M_{\xi},
\end{aligned}
$$

with $\rho_{R}(x, \xi) \triangleq\left(J_{d}-D_{d}\right)(R-\Phi(x, \xi))(x-\xi)$, and consider the submanifold $\mathcal{M} \subset \mathbb{R}^{n} \times \mathbb{R}^{n}$ defined as

$$
\mathcal{M} \triangleq\left\{(x, \xi) \in \mathbb{R}^{n} \times \mathbb{R}^{n}: \nabla_{\xi} H_{d}(x, \xi)=0\right\} .
$$

Proposition 4: Consider the PCH system (1). Let $J_{d}=-J_{d}^{\top}$ and $D_{d}=D_{d}^{\top} \geq 0$. Let $P: \mathbb{R}^{n} \rightarrow \mathbb{R}^{n \times n}$ be an algebraic solution of (3). Suppose that there exist positive definite matrices $M_{x}, M_{\xi}$ and $R$ and a non-empty set $\tilde{\Omega}$, containing the origin, such that

$$
\operatorname{span}\left\{\rho_{R}(x, \xi)\left(\nabla_{x} H_{d}\right)^{\top} M_{x}+M_{x}\left(\nabla_{x} H_{d}\right) \rho_{R}(x, \xi)^{\top}\right\}
$$

is a subset of $\operatorname{span}\left\{D_{d}\right\}$ for all $(x, \xi) \notin \mathcal{M}$ and

$$
\frac{\rho_{R}(x, \xi)}{\left\|\nabla_{x} H_{d}\right\|^{2}}\left(\nabla_{x} H_{d}\right)^{\top} M_{x}+M_{x}\left(\nabla_{x} H_{d}\right) \frac{\rho_{R}(x, \xi)^{\top}}{\left\|\nabla_{x} H_{d}\right\|^{2}}+D_{d} \geq 0
$$

for all $(x, \xi) \in \mathcal{M} \cap \tilde{\Omega}$. Then there exist $\bar{\varepsilon}>0$ and a nonempty set $\Omega \subset \mathbb{R}^{n} \times \mathbb{R}^{n}$ such that system (1) in closed-loop with

$$
\begin{aligned}
\dot{\xi}= & -K \nabla_{\xi} H_{d}+\Gamma_{\xi}^{\top} \nabla_{x} H_{d}, \\
u= & \left(g^{\top} g\right)^{-1} g^{\top}\left[\left(J_{d}-D_{d}\right) \nabla_{x} H_{d}-\varepsilon \Gamma_{x} \nabla_{x} H_{d}\right. \\
& \left.-\Gamma_{\xi} \nabla_{\xi} H_{d}-(J-D) \nabla H\right]-\kappa g^{\top} \nabla_{x} H_{d}
\end{aligned}
$$

can be written in $\mathrm{PCH}$ form for all $(x, \xi) \in \Omega$ and for any $\varepsilon \in(0, \bar{\varepsilon})$.

Proof. By definition of $\tau$ in (14) and recalling that $(x, \xi)=$ $(0,0)$ is a strict (local) minimizer of $H_{d}$, it follows that $\tau(0,0)=0$ and $\tau(x, \xi)>0$ for all $(x, \xi)$ in a neighborhood $\hat{\Omega}$ of the origin. Moreover, noting that each entry of the $n \times n$ matrices at numerator and the scalar function at denominator of $\Gamma_{x}$ and $\Gamma_{\xi}$ are, by (9), locally quadratic functions of $(x, \xi)$ implies that the matrices in (15) are continuous for all $(x, \xi) \in \hat{\Omega} \backslash\{0\}$ and bounded for all $(x, \xi) \in \hat{\Omega}$ by the properties of $\tau$. Then note that $\rho_{R}$ is given by

$\varepsilon \Gamma_{x} \nabla_{x} H_{d}+\Gamma_{\xi} \nabla_{\xi} H_{d}=\frac{\rho_{R}}{\tau}\left(\varepsilon\left\|\nabla_{x} H_{d}\right\|_{M_{x}}^{2}+\left\|\nabla_{\xi} H_{d}\right\|_{M_{\xi}}^{2}\right)$.

Therefore, by (20) and recalling the arguments in the proof of Proposition 2, the closed-loop system (1)-(19) can be written as

$$
\begin{aligned}
\dot{x} & =\left(J_{d}-D_{d}\right) \nabla_{x} H_{d}-\varepsilon \Gamma_{x} \nabla_{x} H_{d}-\Gamma_{\xi} \nabla_{\xi} H_{d}+g(x) v, \\
\dot{\xi} & =-K \nabla_{\xi} H_{d}+\Gamma_{\xi}^{\top} \nabla_{x} H_{d},
\end{aligned}
$$


for all $(x, \xi) \in \hat{\Omega}$. Let now $\Gamma_{x}^{s}$ and $\Gamma_{x}^{a}$ denote the symmetric and the antisymmetric parts of the matrix $\Gamma_{x}$, respectively, namely $\Gamma_{x}^{s}=\operatorname{sym}\left\{\Gamma_{x}\right\}=\left(\Gamma_{x}+\Gamma_{x}^{\top}\right) / 2$ and $\Gamma_{x}^{a}=\operatorname{skew}\left\{\Gamma_{x}\right\}=\left(\Gamma_{x}-\Gamma_{x}^{\top}\right) / 2$. Then, by the assumption on (17), there exist a sufficiently small $\bar{\varepsilon}$ and a non-empty set $\mathcal{W}_{1} \subseteq \hat{\Omega} \backslash \mathcal{M}$ such that $D_{d}+\varepsilon \Gamma_{x}^{s} \geq 0$ for all $\varepsilon \in(0, \bar{\varepsilon})$ and for all $(x, \xi) \in \mathcal{W}_{1}$. Moreover, the matrix $D_{d}+\varepsilon \Gamma_{x}^{s}$, which reduces to the left-hand side of (18) in $\mathcal{M}$, is positive semidefinite by assumption for all $(x, \xi) \in \mathcal{W}_{2} \triangleq(\mathcal{M} \cap \tilde{\Omega}) \cap \hat{\Omega}$. Thus, letting $z=\left[x^{\top}, \xi^{\top}\right]^{\top}$, system (21) can be written in port-controlled Hamiltonian form as

$$
\dot{z}=\left(J_{z}(z)-D_{z}(z)\right) \nabla_{z} H_{d}+G(z) v,
$$

with

$$
J_{z}=\left[\begin{array}{cc}
\left(J_{d}-\varepsilon \Gamma_{x}^{a}\right) & -\Gamma_{\xi} \\
\Gamma_{\xi}^{\top} & 0
\end{array}\right], D_{z}=\left[\begin{array}{cc}
\left(D_{d}+\varepsilon \Gamma_{x}^{s}\right) & 0 \\
0 & K
\end{array}\right] \text {, }
$$

and $G(z)=\left[g(x)^{\top} 0\right]^{\top}$, for all $(x, \xi) \in \mathcal{W}_{1} \cup \mathcal{W}_{2}$, thus proving the claim.

Note that the constant $\varepsilon$ is employed to distribute, in equation (20), the contribution of the matrix coefficients $\Gamma_{x}$ and $\Gamma_{\xi}$, which do not play a symmetric role in the definition of extended PCH system (22). In fact, $\Gamma_{\xi}$ only contributes to the interconnection matrix $J_{z}$ that does not need to satisfy any additional requirement apart from being skew-symmetric, while $\Gamma_{x}^{s}$ appears in $D_{z}$ that, on the other hand, must be positive semi-definite.

Corollary 1: Consider the PCH system (1). Let $J_{d}=-J_{d}^{\top}$ and $D_{d}=D_{d}^{\top}>0$. Let $P: \mathbb{R}^{n} \rightarrow \mathbb{R}^{n \times n}$ be an algebraic solution of (3). Suppose that there exists a non-empty set $\tilde{\Omega}$, containing the origin, such that (18) holds for all $(x, \xi) \in \mathcal{M} \cap$ $\tilde{\Omega}$. Then there exist $\bar{\varepsilon}>0$ and a non-empty set $\Omega \subset \mathbb{R}^{n} \times \mathbb{R}^{n}$ such that the closed-loop system (1)-(19) can be written in $\mathrm{PCH}$ form for all $(x, \xi) \in \Omega$ and for any $\varepsilon \in(0, \bar{\varepsilon})$.

Proof. The claim follows immediately from the arguments employed in the proof of Proposition 4 and by noting that $D_{d}$ is positive definite.

Remark 6: As suggested by the proof of Proposition 4, the matrix $\varepsilon \Gamma_{x}$, which perturbs the desired interconnection and damping matrices, can be rendered arbitrarily small in some non-empty set containing the equilibrium by selecting a sufficiently small $\varepsilon$. Moreover, the neighborhood $\mathcal{W}_{1}$ and the constant $\bar{\varepsilon}$ are strongly related since the set $\mathcal{W}_{1}$ can be enlarged by considering increasingly smaller values of $\bar{\varepsilon}$. On the other hand, for all $(x, \xi) \in \mathcal{M}$, the matrix

$$
\varepsilon \Gamma_{x}=\varepsilon \frac{\rho(x, \xi)}{\tau(x, \xi)}\left(\nabla_{x} H_{d}\right)^{\top} M_{x}=\frac{\rho(x, \xi)}{\left\|\nabla_{x} H_{d}\right\|_{M_{x}}^{2}}\left(\nabla_{x} H_{d}\right)^{\top} M_{x}
$$

does not depend upon $\varepsilon$, hence it cannot be rendered arbitrarily small for sufficiently small $\varepsilon$, motivating the rationale behind inequality (18).

We are now in the position to state the following result, which shows that if the matching equation (3) does not admit a classical solution for a desired interconnection and damping structure but it does admit an algebraic solution, then there exists a classical, static, solution to a matching equation corresponding to the interconnection and damping structure given by the sum of the desired matrices and some continuous functions of $x$, which vanish at the desired equilibrium.

Theorem 1: Consider the PCH system (1). Let $J_{d}=-J_{d}^{\top}$ and $D_{d}=D_{d}^{\top}>0$ and suppose that the matching equation (3) admits an algebraic solution $P$. Then there exist continuous matrix-valued functions $J_{s}: \mathbb{R}^{n} \rightarrow \mathbb{R}^{n \times n} J_{s}(0)=0$, $J_{s}(x)=-J_{s}(x)^{\top}$, and $D_{s}: \mathbb{R}^{n} \rightarrow \mathbb{R}^{n \times n}, D_{s}(0)=0$, $D_{s}(x)=D_{s}(x)^{\top}$, and an energy function $H_{s}: \mathbb{R}^{n} \rightarrow \mathbb{R}$ that solves the partial differential equations (3) associated to the interconnection and damping structure given by $J_{d}-J_{s}$ and $D_{d}+D_{s}$, respectively.

Proof. By definition of algebraic solution

$$
\begin{aligned}
g(x)^{\perp}(J-D) \nabla H & =g(x)^{\perp}(J-D)(L+N(x) x) \\
& =g(x)^{\perp}\left(J_{d}-D_{d}\right)(N(x)+P(x)) x \\
& =g(x)^{\perp}\left(J_{\varepsilon}-D_{\varepsilon}\right) \nabla_{x} H_{d}-\Gamma_{\xi} \nabla_{\xi} H_{d},
\end{aligned}
$$

with $J_{\varepsilon}=J_{d}-\varepsilon \Gamma_{x}^{a}(x, \xi)$ and $D_{\varepsilon}=D_{d}+\varepsilon \Gamma_{x}^{s}(x, \xi)$, where the last equation is obtained by (5), and the results in Proposition 4 and Corollary 1. Recalling now that $(x, \xi)=(0,0)$ is a strict local minimizer of $H_{d}$, it follows that $\left.\nabla_{\xi} H_{d}\right|_{(x, \xi)=(0,0)}=0$ and $\left.\nabla_{\xi \xi} H_{d}\right|_{(x, \xi)=(0,0)}>0$. Thus, by the Implicit Function Theorem there exists a continuously differentiable function $\gamma: \mathbb{R}^{n} \rightarrow \mathbb{R}^{n}$ such that $\left.\nabla_{\xi} H_{d}\right|_{\xi=\gamma(x)}=0$. Then, by [4, Prop. 3], the function $H_{s}(x) \triangleq H_{d}(x, \gamma(x))$ solves the matching equation (3) corresponding to the interconnection $J_{d}-\varepsilon \Gamma_{x}^{a}(x, \gamma(x))$ and the damping $D_{d}+\varepsilon \Gamma_{x}^{s}(x, \gamma(x))$. As discussed in Remark 6 the matrix $\varepsilon \Gamma_{x}(x, \gamma(x))$ does not depend upon $\varepsilon$. Moreover, by the properties of $\tau$ it follows that $\left\|\nabla_{x} H_{d}(x, \gamma(x))\right\|_{M_{x}}^{2}$, namely the denominator of $\varepsilon \Gamma_{x}(x, \gamma(x))$, is (locally) positive definite and given by the sum of quadratic functions of $x$ and higher order terms. Finally, the choice $R=\Phi(0, \gamma(0))=0$, which renders the function $H_{d}(x, \gamma(x))$ (locally) positive definite in the restricted $x$ state-space, is such that each entry of the matrix at the numerator of $\varepsilon \Gamma_{x}(x, \gamma(x))$ has at least cubic terms in the variable $x$. Therefore, the matrix $\varepsilon \Gamma_{x}(x, \gamma(x))$ is continuous for all $x \in \mathbb{R}^{n}$ and such that $\varepsilon \Gamma_{x}(0, \gamma(0))=0$, concluding the proof.

Remark 7: The results of Theorem 1 can be interpreted as follows. Given any positive constant $\mu>0$ there exists a nonempty compact set $\mathcal{C}$, containing the origin, such that the existence of a solution to the dynamic matching equation implies the existence of a solution to the static matching equation corresponding to an interconnection $J_{e}$ and damping $D_{e}$ structure satisfying $\sup _{i, j \in\{1, \ldots, n\}, x \in \mathcal{C}}\left\|\left(J_{d}(x)\right)_{i, j}-\left(J_{e}(x)\right)_{i, j}\right\|<\mu$ and $\sup _{i, j \in\{1, \ldots, n\}, x \in \mathcal{C}}\left\|\left(D_{d}(x)\right)_{i, j}-\left(D_{e}(x)\right)_{i, j}\right\|<\mu$. In other words, the solution to the dynamic matching equation implies the existence of a local solution to the static matching equation corresponding to an interconnection and damping structure arbitrarily close to the desired one.

Remark 8: The nature of the results in Theorem 1 is intrinsically local by definition of $\gamma$ as in the Implicit Function Theorem. Hence, even though Theorem 1 shows that there 
exists a (local) classical static solution to an IDA-PBC problem corresponding to interconnection and damping matrices arbitrarily close to the desired ones, it may be preferable to implement the dynamic control law (19), in place of the static feedback provided by Theorem 1, since the latter may enforce a smaller domain of stability of the assigned equilibrium.

Finally, an alternative result that allows to preserve the $\mathrm{PCH}$ structure, provided an additional technical assumption on the algebraic solution $P$ is satisfied and if the matrix $R$ is allowed to vary over time, is presented.

Proposition 5: Consider the PCH system (1). Let $J_{d}=-J_{d}^{\top}$ and $D_{d}=D_{d}^{\top} \geq 0$. Let $P$ be an algebraic solution of (3) such that $\Phi(x, \xi)=\Phi(x, \xi)^{\top}>0$ in some non-empty open neighborhood $\Omega$ of the origin. Let $R(t)=\Phi(x(t), \xi(t))$ for all $t \geq 0$. Then the closed-loop system (1)-(11) reduces to $\dot{z}=\left(J_{z}-D_{z}\right) \nabla_{z} H_{d}$ for all $(x, \xi) \in \Omega$, with $J_{z}=$ $\operatorname{blockdiag}\left(J_{d}, 0\right)$ and $D_{z}=\operatorname{blockdiag}\left(D_{d}, K\right)$.

Proof. The claim follows directly recalling the arguments in the proof of Proposition 2, namely the structure of system (12).

Remark 9: Despite the fact that the closed-loop system (1)-(11) possesses a PCH structure, an additional condition is required to ensure that $\dot{H}_{d} \leq y_{d}^{\top} v$, due to the dependency of the energy function $H_{d}$ on time via the matrix $R$. In particular, following the same arguments as those in the proof of Proposition 2 , the condition $\Psi^{\top} R^{-1} \dot{\Phi} R^{-1} \Psi<2\left(N^{\top}+P\right) D_{d}(N+P)$ implies the existence of $\bar{K}=\bar{K}^{\top}$ such that $\dot{H}_{d}(t, x, \xi) \leq y_{d}^{\top} v$ for all $K>\bar{K}$.

\section{LINEAR SYSTEMS}

In this section we specialize the results to the case of linear, time-invariant, $\mathrm{PCH}$ systems, namely

$$
\dot{x}=(J-D) \nabla H+B u,
$$

where the energy $H$ is defined as in (4), with the function $h$ identically equal to zero, and $B \in \mathbb{R}^{n \times m}$ has rank $m$. The proposed approach leads, under standard assumptions and for a specific choice of the design parameters, exactly to the classical solution of the IDA-PBC problem for linear systems. The matching equation (3) reduces to

$$
B^{\perp}\left[(J-D)(L+\bar{H} x)-\left(J_{d}-D_{d}\right)(\bar{H}+\bar{P}) x\right]=0,
$$

for all $x \in \mathbb{R}^{n}$, where the matrix $B^{\perp} \in \mathbb{R}^{(n-m) \times n}$ of rank $n-m$ is such that $B^{\perp} B=0$. Note that in the linear case the desired energy function becomes $H_{d}(z)=\frac{1}{2} z^{\top} \bar{H}_{d} z$.

Corollary 2: Consider the PCH system (23). Let $J_{d}=-J_{d}^{\top}$ and $D_{d}=D_{d}^{\top} \geq 0$. Let $\bar{P}$ be a solution of (24) such that $\bar{H}+\bar{P}>0$ and consider the dynamic control law

$$
\begin{aligned}
\dot{\xi}= & -K \nabla_{\xi} H_{d}, \\
u= & \left(B^{\top} B\right)^{-1} B^{\top}\left[\left(J_{d}-D_{d}\right)((\bar{H}+\bar{P}) x+R(x-\xi))\right. \\
& -(J-D)(L+\bar{H} x)]-\kappa B^{\top} \nabla_{x} H_{d} .
\end{aligned}
$$

Let $R=0$, then the point $(x, \xi)=(0,0)$ is a stable equilibrium of the closed-loop system (23)-(25) for all $K>0$ and $\kappa \geq 0$. If, in addition, (23)-(25) is zero-state detectable with respect to the output $y_{d}=B^{\top}(\bar{H}+\bar{P}) x$, then, for any $\kappa>0$, $x(t)$ asymptotically converges to the origin. Moreover, (25) reduces to the classical (static) solution of the IDA-PBC design problem for linear PCH systems.

Proof. Note that, since $R=0,(\bar{H}+\bar{P}) x=\nabla_{x} H_{d}$, and the closed-loop system becomes, with $\kappa=0$, by definition of $u$ in the second of equations (25) and by definition of $\bar{P}$ in (24), $\dot{x}=\left(J_{d}-D_{d}\right) \nabla_{x} H_{d}, \dot{\xi}=-K \nabla_{\xi} H_{d}$. Then, $\bar{H}_{d}=$ blockdiag $(\bar{H}+\bar{P}, 0)$, which allows to prove the first claim by recalling that $\bar{H}+\bar{P}$ is positive definite by assumption. The dynamics of $\xi$ in (25) become $\dot{\xi}=0$, hence the second equation reduces to the classical static state-feedback solving the IDA-PBC problem for system (23).

The statement of Corollary 2 entails that stability of the zero equilibrium of the extended system (23)-(25) is enforced without additional restrictions on the choice of the matrix $R$. The following proposition proves that the same result is achieved by showing that system (23) in closed-loop with an alternative dynamic control law exhibits a PCH structure.

Proposition 6: Consider the $\mathrm{PCH}$ system (23). Let $J_{d}=$ $-J_{d}^{\top}$ and $D_{d}=D_{d}^{\top} \geq 0$. Let $\bar{P}$ be a solution of (24) and consider the dynamic control law

$$
\begin{aligned}
\dot{\xi}= & \left(J_{d}+D_{d}\right) \nabla_{x} H_{d}-K \nabla_{\xi} H_{d}, \\
u= & \left(B^{\top} B\right)^{-1} B^{\top}\left[\left(J_{d}-D_{d}\right)((\bar{H}+\bar{P}) x)\right. \\
& (J-D)(L+\bar{H} x)]-\kappa B^{\top} \nabla_{x} H_{d} .
\end{aligned}
$$

Then the closed-loop system (23)-(26) can be written in $\mathrm{PCH}$ form.

Proof. Note that, setting $v=0$, according to Corollary 2 the closed-loop system (23)-(25) can be written as

$$
\begin{aligned}
& \dot{x}=\left(J_{d}-D_{d}\right)(\bar{H}+\bar{P}) x, \\
& \dot{\xi}=\left(J_{d}+D_{d}\right) \nabla_{x} H_{d}-K \nabla_{\xi} H_{d} .
\end{aligned}
$$

Consider now the gradient of the auxiliary function $H_{d}$ as in (9) which reduce, in the linear case, to

$$
\begin{aligned}
\nabla_{x} H_{d} & =(\bar{H}+\bar{P}) x+R(x-\xi), \\
\nabla_{\xi} H_{d} & =-R(x-\xi) .
\end{aligned}
$$

Note now that $(\bar{H}+\bar{P}) x=\nabla_{x} H_{d}+\nabla_{\xi} H_{d}$, which, substituted into the closed-loop system (27), proves that the latter may be written as the PCH system $\dot{z}=(\hat{J}-\hat{D}) \nabla_{z} H_{d}$, with

$$
\hat{J}=\left[\begin{array}{cc}
J_{d} & \left(J_{d}-D_{d}\right) \\
\left(J_{d}+D_{d}\right) & 0
\end{array}\right]
$$

and $\hat{D}=\operatorname{blockdiag}\left(D_{d}, K\right)$, hence the claim.

\section{APPliCATIONS: MAgNetiC LEVITATED BALL AND A THIRD ORDER FOOD-CHAIN SYSTEM}

We motivate the constructive IDA-PBC approach presented in this paper by designing a state-feedback controller for a magnetically levitated ball and a third order food-chain system. In both cases, the control design is based on the theory discussed in Sections IV and V. 


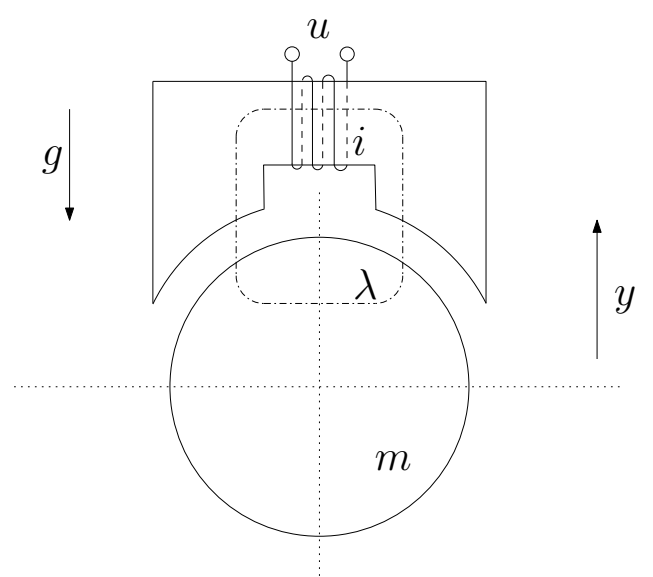

Figure 1. A schematic representation of the magnetically levitated system

\section{A. Magnetic levitated ball}

1) Model: A magnetic levitated system which consists of an iron ball of mass $m$, in a vertical magnetic field created by a single electromagnet is shown in Figure 1. The flux $\lambda$ generated by the magnet is assumed to be unsaturated, i.e. $\lambda=\eta(\theta) i$ where $\theta$ is the difference between the position of the center of the ball and its nominal position, with the $\theta$-axis oriented downward, $i$ is the current flowing through a coil of resistance $r, \eta(\theta)$ denotes the value of the inductance. By combining Kirchoff's voltage law and Newton's second law and by considering an approximation for the inductance, the dynamics of the system may be described by the equations [21]

$$
\begin{aligned}
\dot{\lambda}+r i & =u, \\
m \ddot{\theta} & =F-m g,
\end{aligned}
$$

where $F$ is the force induced by the electromagnet, which is proportional to the derivative of the inductance with respect to the displacement $\theta$ and to the square of the current $i$, i.e. $F \propto(\partial \eta / \partial \theta) i^{2}$, while $g$ denotes the acceleration due to gravity. In particular, borrowing the approximation from [21] we let $\eta(\theta)=k /(1-\theta)$, where $k$ is some positive constant that depends on the number of coil turns, in the domain $\theta \in(-\infty, 1)$. It has been shown in [21] that the system (29) can be written in $\mathrm{PCH}$ form with the state variable $x=[\lambda, \theta, m \dot{\theta}]$, and the Hamiltonian function

$$
H(x)=\frac{1}{2 k}\left(1-x_{2}\right) x_{1}^{2}+\frac{1}{2 m} x_{3}^{2}+m g x_{2},
$$

yielding

$$
\dot{x}=\underbrace{\left[\begin{array}{ccc}
-r & 0 & 0 \\
0 & 0 & 1 \\
0 & -1 & 0
\end{array}\right]}_{J-D} \frac{\partial H}{\partial x}(x)+\left[\begin{array}{l}
1 \\
0 \\
0
\end{array}\right] u .
$$

The equilibrium to stabilize is $x_{*}=\left[\sqrt{2 k m g}, x_{2^{*}}, 0\right]$, i.e. a constant position of the ball.

2) Controller design: It has been shown in [21] that the energy shaping problem cannot be solved for system (31) with $H$ as in (30), i.e. the equilibrium $x_{*}$ cannot be stabilized by assigning a desired energy function $H_{d}(x)$ while preserving the interconnection structure of system (31). This negative result may be intuitively explained by noting the lack of coupling between the electrical and the mechanical subsystems resulting from the interconnection matrix $J$ associated to (31), which couples only the velocity with the position. Therefore, an Interconnection and Damping Assignment passivity-based control problem should be solved. It is shown in [21] that selecting the desired interconnection matrix $J_{d}$ such that it couples the flux $x_{1}$ and the velocity $x_{3}$ - while preserving the damping structure of system (30) or removing the damping from the electrical subsystem and adding it to the position yields a system of partial differential equations of the form (3), the solution of which can be provided in closed-form, see [21] for more details.

To illustrate the results presented in Section V we let the desired interconnection and damping structure be defined by the matrices

$$
J_{d}=\left[\begin{array}{ccc}
0 & \alpha & \beta \\
-\alpha & 0 & \gamma \\
-\beta & -\gamma & 0
\end{array}\right], D_{d}=\left[\begin{array}{ccc}
d_{11} & d_{12} & d_{13} \\
d_{12} & d_{22} & d_{23} \\
d_{13} & d_{23} & d_{33}
\end{array}\right]
$$

where $\alpha, \beta, \gamma$ are functions to be defined and $d_{11}, d_{12}, d_{13}, d_{22}, d_{23}$ and $d_{33}$, are such that $D_{d}$ is positive definite. Note that $J_{d}$ is such that the flux $x_{1}$ is coupled with the velocity $x_{3}$, as in [21], and the position $x_{2}$ and, unlike in [21], a positive definite damping matrix is considered. The latter matrix allows to conclude asymptotic stability of the desired equilibrium without the need for the additional passive output injection, namely with $\kappa=0$. The interest in the selection of $J_{d}$ and $D_{d}$ as in (32) lies in the fact that, with this choice, the resulting matching equations (3)

$$
\begin{aligned}
& \left(\alpha+d_{12}\right) \frac{\partial \mathcal{K}}{\partial x_{1}}+d_{22} \frac{\partial \mathcal{K}}{\partial x_{2}}-\left(\gamma-d_{23}\right) \frac{\partial \mathcal{K}}{\partial x_{3}}= \\
& -\left(\alpha+d_{12}\right) \frac{\partial H}{\partial x_{1}}-d_{22} \frac{\partial H}{\partial x_{2}}+\left(\gamma-d_{23}-1\right) \frac{\partial H}{\partial x_{3}} \\
& \left(\beta+d_{13}\right) \frac{\partial \mathcal{K}}{\partial x_{1}}+\left(\gamma+d_{23}\right) \frac{\partial \mathcal{K}}{\partial x_{2}}+d_{33} \frac{\partial \mathcal{K}}{\partial x_{3}}= \\
& -\left(\beta+d_{13}\right) \frac{\partial H}{\partial x_{1}}-\left(\gamma+d_{23}-1\right) \frac{\partial H}{\partial x_{2}}-d_{33} \frac{\partial H}{\partial x_{3}}
\end{aligned}
$$

which have been obtained by letting

$$
g^{\perp}=\left[\begin{array}{lll}
0 & 1 & 0 \\
0 & 0 & 1
\end{array}\right]
$$

do not admit a solution $\mathcal{K}: \mathbb{R}^{3} \rightarrow \mathbb{R}$. However, we show in the following that, by letting the desired interconnection and damping matrices be possibly function of $x$, an algebraic solution can be determined for the above Interconnection and Damping Assignment passivity-based control problem, leading to a dynamic control law of the form (19). Towards this end, and to fit into the theoretical framework introduced in the previous sections, consider the change of coordinates $\tilde{x}=x-x_{*}$. In the new coordinates the gradient of the function 


$$
\begin{gathered}
D_{d} \triangleq\left[\begin{array}{ccc}
c \tilde{x}_{1}^{2} & 0 & 0 \\
0 & \frac{1}{m\left(3 c \tilde{x}_{1}^{2}+4\right)} & \frac{3 m \tilde{x}_{1}-2 k+6 m \tilde{x}_{1 *}}{12 m k\left(3 c \tilde{x}_{1}^{2}+4\right)}-\frac{1}{3 m} \\
0 & \frac{3 m \tilde{x}_{1}-2 k+6 m \tilde{x}_{1 *}}{12 m k\left(3 c \tilde{x}_{1}^{2}+4\right)}-\frac{1}{3 m} & \frac{\left(\tilde{x}_{1}+2 x_{1 *}\right)\left(c \tilde{x}_{1}^{2}+1\right)}{2 k\left(3 c \tilde{x}_{1}^{2}+4\right)}
\end{array}\right] . \\
J_{d} \triangleq\left[\begin{array}{ccc}
\frac{c \tilde{x}_{1}^{2}+1}{m\left(3 c \tilde{x}_{1}^{2}+4\right)} & -\frac{\left(\tilde{x}_{1}+2 x_{1 *}\right)\left(2 c \tilde{x}_{1}^{2}+3\right)}{2 k\left(3 c \tilde{x}_{1}^{2}+4\right)} \\
-\frac{c \tilde{x}_{1}^{2}+1}{m\left(3 c \tilde{x}_{1}^{2}+4\right)} & 0 & \frac{3 m \tilde{x}_{1}+2 k+6 m \tilde{x}_{1 *}}{12 m k\left(3 c \tilde{x}_{1}^{2}+4\right)}+\frac{1}{3 m} \\
\frac{\left(\tilde{x}_{1}+2 x_{1 *}\right)\left(2 c \tilde{x}_{1}^{2}+3\right)}{2 k\left(3 c \tilde{x}_{1}^{2}+4\right)} & -\frac{3 m \tilde{x}_{1}+2 k+6 m \tilde{x}_{1 *}}{12 m k\left(3 c \tilde{x}_{1}^{2}+4\right)}-\frac{1}{3 m} & 0
\end{array}\right] .
\end{gathered}
$$
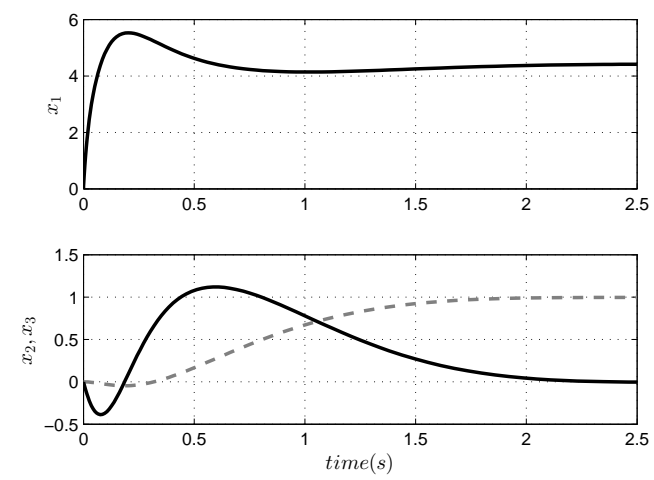

Figure 2. Top graph: Time history of the state $x_{1}(t)$, in the original $x$ coordinates, of the electrical subsystem of (31) in closed-loop with the dynamic control law (11). Bottom graph: Time histories of the states, in the original $x$-coordinates, of the mechanical subsystem of (31), namely $x_{2}(t)$ and $x_{3}(t)$, gray and dark line, respectively.

$H$ in (30) becomes

$$
\nabla H=\left[\begin{array}{c}
\frac{1}{k}\left(1-x_{2^{*}}-\tilde{x}_{2}\right)\left(\tilde{x}_{1}+x_{1^{*}}\right) \\
m g-\frac{1}{2 k}\left(\tilde{x}_{1}+x_{1^{*}}\right)^{2} \\
\frac{1}{m} \tilde{x}_{3}
\end{array}\right],
$$

which yields $L=\left[x_{1^{*}}\left(1-x_{2^{*}}\right) / k \quad 0 \quad 0\right]^{\top} \triangleq\left[\begin{array}{lll}\ell_{1} & 0 & 0\end{array}\right]$ according to the definition of $H$ in (4). Suppose that the desired equilibrium to stabilize is $x_{2^{*}}=1$, then a nonsymmetric matrix $N$, as in Definition 2, can be defined:

$$
N(\tilde{x})=\left[\begin{array}{ccc}
0 & -\frac{1}{k}\left(\tilde{x}_{1}+x_{1^{*}}\right) & 0 \\
-\frac{1}{k}\left(\frac{1}{2} \tilde{x}_{1}+x_{1^{*}}\right) & 0 & 0 \\
0 & 0 & \frac{1}{m}
\end{array}\right] .
$$

Considering the equation (5) introduced in Definition 2 together with the comments contained in Remark 1 , let $D_{d}$ be defined as in (35) which is locally positive definite provided $c>0$, and $J_{d}$ be defined as in (36).
It is now easy to show that the matrix-valued function

$$
P(\tilde{x}) \triangleq\left[\begin{array}{ccc}
2 & 1 & 1 \\
1 & 2+c \tilde{x}_{1}^{2} & 1 \\
1 & 1 & 2
\end{array}\right]
$$

solves the algebraic equation (5) in the form discussed in details in Remark 1 , namely it is such that $g(\tilde{x})^{\perp}[(J-$ $\left.D) N(\tilde{x})-\left(J_{d}-D_{d}\right) P(\tilde{x})\right]=0$. The above under-determined system of equations has been solved by fixing a desired structure for one of the independent variables, namely $P$, and consequently computing $J_{d}$ and $D_{d}$ by means of standard software performing symbolic computations. Note that $P$ in (35) depends on the parameter $c>0$. Moreover, item $(i)$ of Definition 2 is satisfied by the function $P$ in (35). Interestingly, the mapping $x \mapsto P(x) x$ is not the gradient of any scalar positive definite function. In fact, the Jacobian matrix

$$
\frac{\partial P(x) x}{\partial x}=\left[\begin{array}{ccc}
2 & 1 & 1 \\
1+2 c x_{1} x_{2} & c x_{1}^{2}+2 & 1 \\
1 & 1 & 2
\end{array}\right]
$$

is not symmetric for all $x \in \mathbb{R}^{3}$, whenever $c$ is strictly positive, which is on the other hand the requirement needed to enforce (local) positive semi-definiteness of the damping matrix $D_{d}$.

In the following numerical simulation the objective is to stabilize the ball at $x_{2 *}=1$. Let $r=m=k=1$ and consider the damping and interconnection structure in (35) and (36), respectively. The dynamic control law $u$ is obtained according to (19) with $c=0.1$ and $\varepsilon=10^{-3}$, which renders the origin of the $\tilde{x}$-coordinates a strict local minimizer of the extended desired energy $H_{d}$ defined in (8), and $K=\operatorname{diag}(\{10,10,10\})$. Note that the control law (19) preserves the Port-controlled Hamiltonian structure in the closed-loop extended system, which allows, together with the fact that the damping matrix $D_{d}$ is positive definite, to enforce local asymptotic stability of the origin in the $\tilde{x}$-coordinates without the additional output injection, namely with $\kappa=0$ in the second equation of (19).

The top graph of Figure 2 shows the time history of the state, in the original $x$-coordinates, of the electrical subsystem of (31) for the initial conditions $x(0)=[0,0,0]$ and $\xi(0)=$ $[10,-20,10]$ in closed-loop with the dynamic control law (11). 

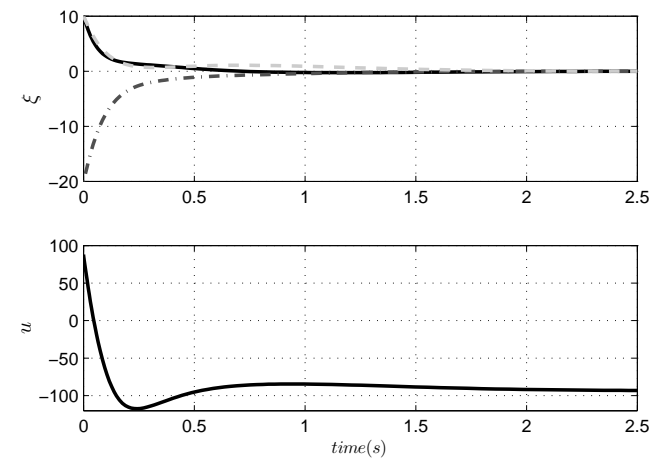

Figure 3. Top graph: Time histories of the dynamic extension $\xi_{1}(t)$ (solid), $\xi_{2}(t)$ (dash-dotted), $\xi_{3}(t)$ (dashed). Bottom graph: Time history of the control input $u$ in (11).

The bottom graph, on the other hand, displays the time histories of the states, in the original $x$-coordinates, of the mechanical subsystem of (31), namely $x_{2}(t)$ and $x_{3}(t)$. Finally, Figure 3 shows the time histories of the states of the dynamic extension, $\xi(t)$, and of the control law $u$ in (11), top and bottom graph, respectively.

\section{B. Third order food-chain system}

1) Model: Consider the normalized third order food-chain system from [13] for which an IDA-PBC controller has been designed in [20]. In this food-chain system the variable $x_{i}$ for $i=1,2,3$ represents the population of the $i$-th species involved in the system. The growth rates of the population is given by

$$
\begin{aligned}
& \dot{x}_{1}=f_{1}(x)-x_{1}, \\
& \dot{x}_{2}=-f_{1}(x)+f_{2}(x)-x_{2}, \\
& \dot{x}_{3}=-f_{2}(x)-x_{3}+u .
\end{aligned}
$$

The functions $f_{1}(x)$ and $f_{2}(x)$ describe the predation mechanism, such as for instance Lotka-Volterra mechanism. It is assumed that the rate of predation upon the prey is proportional to the rate at which the predators and prey meet i.e. $f_{1}(x)=x_{1} x_{2}$ and $f_{2}(x)=x_{2} x_{3}$. The terms $-x_{1},-x_{2},-x_{3}$ represent the rate of loss of predators due to natural death or emigration and the control action $u$ is the rate of preys being added to the system. It is clear that the food-chain system (38) can be written in a PCH form with $H=x_{1}+x_{2}+x_{3}$, $g=[0,0,1]^{\top}$ and

$J=\left[\begin{array}{ccc}0 & x_{1} x_{2} & 0 \\ -x_{1} x_{2} & 0 & x_{2} x_{3} \\ 0 & -x_{2} x_{3} & 0\end{array}\right], D=\left[\begin{array}{ccc}x_{1} & 0 & 0 \\ 0 & x_{2} & 0 \\ 0 & 0 & x_{3}\end{array}\right]$.

The evolution of the system is restricted to the positive orthant with $u \geq 0$. The control objective is to stabilize a given nonzero equilibrium $\bar{x} \in \mathcal{R}_{+}^{3}$. Note that the achievable equilibria are $\bar{x}=\left[\bar{x}_{1}, \bar{x}_{2}, \bar{x}_{1}\right]^{\top}=\left[x_{1^{*}}, 1,1+x_{1^{*}}\right]^{\top}$, with $x_{1^{*}}>0$.

2) Controller design: It has been shown in [20] that the matching equation PDE cannot be solved when $J_{d}=J$ and with a $D_{d}>0$ because the distribution spanned by the vector fields defined by the column vectors obtained from the first 2 rows of $J(x)-D(x)$ is not involutive. To overcome this limitation the damping of the closed-loop system is modified to be $D_{d}=\operatorname{diag}\left(0,0, x_{3}\right)$ which is clearly positive semidefinite. To address this IDA-PBC problem and illustrate the results presented in Section IV consider a shift of coordinates $\tilde{x}=x-\bar{x}$. The dynamics of the system $\dot{\tilde{x}}$ in the new coordinates is described by the matrix

$$
\underbrace{\left[\begin{array}{ccc}
0 & \tilde{x}_{1} \tilde{x}_{2}+\tilde{x}_{2} x_{1 *} & 0 \\
-\tilde{x}_{1} \tilde{x}_{2}-\tilde{x}_{2} x_{1^{*}} & -\tilde{x}_{1} & \tilde{x}_{2} \tilde{x}_{3}+\tilde{x}_{2} x_{1^{*}}+\tilde{x}_{3} \\
0 & -\tilde{x}_{2} \tilde{x}_{3}-\tilde{x}_{2} x_{1^{*}}-\tilde{x}_{3} & -\tilde{x}_{2}-\tilde{x}_{3}
\end{array}\right]}_{J(x)-D(x)},
$$

$g=[0,0,1]^{\top}, \tilde{H}=\tilde{x}_{1}+\tilde{x}_{2}+\tilde{x}_{3}$ and $\tilde{u}=u-2\left(x_{1^{*}}+1\right)$. Let the desired interconnection and damping matrices be

$J_{d}(x)=\left[\begin{array}{ccc}0 & \alpha & \beta \\ -\alpha & 0 & \gamma \\ -\beta & -\gamma & 0\end{array}\right], D_{d}(x)=\left[\begin{array}{ccc}d_{1} & 0 & 0 \\ 0 & d_{2} & 0 \\ 0 & 0 & d_{3}\end{array}\right]$.

where $d_{i}>0$ for $i=1,2,3$, so that $D_{d}$ is positive definite. The structure of $J_{d}$ and $D_{d}$ is motivated by the interconnection and damping matrices of the original system and by noting that the resulting matching equation (38) that are obtained by letting

$$
g^{\perp}=\left[\begin{array}{lll}
1 & 0 & 0 \\
0 & 1 & 0
\end{array}\right],
$$

do not admit a solution $\mathcal{K}: \mathcal{R}^{3} \rightarrow R$. Note that the achievable equilibrium $[0,0,0]^{\top}$ satisfies the condition $g^{\perp}\left[\left(J_{0}-\right.\right.$ $\left.\left.D_{0}\right) L\right]=0$ with $L=[1,1,1]^{\top}$ and that the matrix $N$ in Definition 2 is the zero matrix. To find a matrix-valued function $P$ that satisfies items $(i)$ and (ii) of Definition 2, let $D_{d}(x) \triangleq I$ and $J_{d}$ be defined to be

$\left[\begin{array}{ccc}0 & 1 & -\frac{2 \tilde{x}_{2}+x_{1^{*}}+1}{\tilde{x}_{2}+1} \\ -1 & 0 & \frac{3 \tilde{x}_{2}+x_{1^{*}}+2}{\tilde{x}_{2}+1} \\ -\frac{2 \tilde{x}_{2}+x_{1 *}+1}{\tilde{x}_{2}+1} & -\frac{3 \tilde{x}_{2}+x_{1 *}+2}{\tilde{x}_{2}+1} & 0\end{array}\right]$.

It is easy to show that the matrix-valued function $P$ in (39) is a solution of the algebraic matching equation (5), namely $g(\tilde{x})^{\perp}\left[(J-D) L-\left(J_{d}-D_{d}\right) P(\tilde{x})\right]=0$ and satisfies item $(i)$ of Definition 2. The mapping $\tilde{x} \mapsto P\left(\tilde{x}_{2}\right) \tilde{x}$ is not the gradient of any positive definite scalar function. This can be inferred by noting that the Jacobian matrix of $P\left(\tilde{x}_{2}\right) \tilde{x}$ is not symmetric for all $x \in \mathbb{R}^{3}$.

The numerical simulations of the third order food-chain system have been performed to stabilize the population at $\bar{x}=[1,1,2]$, i.e. $x_{1^{*}}=1$. The dynamic control law (19) is obtained with $\kappa=0$ and $\epsilon=0.01$, where $\kappa$ and $\varepsilon$ are the parameters introduced in Section V. The control law renders the origin of the extended desired system in the $\tilde{x}$ coordinates a strict local minimizer while preserving the portcontrolled Hamiltonian structure of the closed-loop system. Local asymptotic stability of the origin of the extended system in the $\tilde{x}$-coordinates is enforced since the damping matrix $D_{d}$ is positive definite for all $\tilde{x} \in \mathcal{R}^{3}$.

Figure 4 shows the time histories of the states namely $x_{1}, x_{2}$ and $x_{3}$ in the original $x$-coordinates for the initial conditions $x(0)=[2,2,4]$ and $\xi(0)=[10,10,12]$ with the dynamic 


$$
\begin{array}{r}
\tilde{x}_{2} x_{1^{*}}+\tilde{x}_{1} \tilde{x}_{2}=-+d_{1}\left(\frac{\partial \mathcal{K}}{\partial \tilde{x}_{1}}+1\right)+\alpha\left(\frac{\partial \mathcal{K}}{\partial \tilde{x}_{2}}+1\right)+\beta\left(\frac{\partial \mathcal{K}}{\partial \tilde{x}_{3}}+1\right) \\
-\tilde{x}_{1}+\tilde{x}_{3}-\tilde{x}_{1} \tilde{x}_{2}+\tilde{x}_{2} \tilde{x}_{3}=-d_{2}\left(\frac{\partial \mathcal{K}}{\partial \tilde{x}_{2}}+1\right)-\alpha\left(\frac{\partial \mathcal{K}}{\partial \tilde{x}_{1}}+1\right)+\gamma\left(\frac{\partial \mathcal{K}}{\partial \tilde{x}_{3}}+1\right), \\
P=\left[\begin{array}{ccc}
\frac{2+4 \tilde{x}_{2}+\tilde{x}_{2}^{2}+x_{1^{*}}}{\tilde{x}_{2}+1} & \frac{4+\tilde{x}_{2}\left(8+x_{1^{*}}\right)+2 \tilde{x}_{2}^{2}+3 x_{1^{*}}}{\tilde{x}_{2}+1} \\
\frac{2+4 \tilde{x}_{2}+\tilde{x}_{2}^{2}+x_{1^{*}}}{\tilde{x}_{2}+1} & \frac{6+22 \tilde{x}_{2}+23 \tilde{x}_{2}^{2}+5 \tilde{x}_{2}^{3}++5 x_{2}^{2} x_{1^{*}}+x_{2} x_{1^{*}}^{2}+18 x_{2} x_{1^{*}}+9 x_{1^{*}}+3 x_{1^{*}}^{2}}{\left(\tilde{x}_{2}+1\right)^{2}} \\
1 & \frac{4+\tilde{x}_{2}\left(8+x_{1^{*}}\right)+2 \tilde{x}_{2}^{2}+3 x_{1^{*}}}{\tilde{x}_{2}+1}
\end{array}\right]
\end{array}
$$
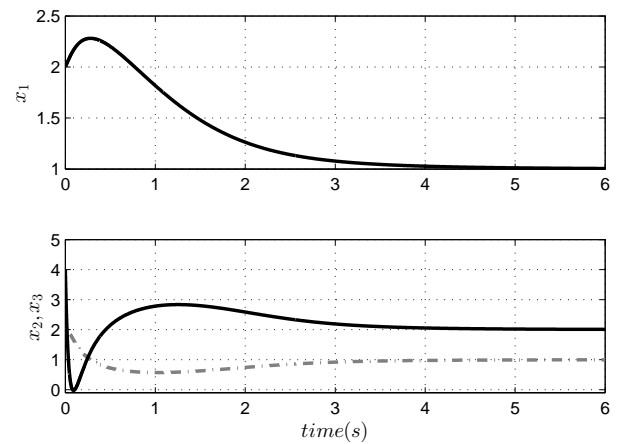

Figure 4. Top graph: Time history of the state, $x_{1}(t)$ in the original $x$ coordinates, of the food-chain system (40) in closed-loop with the dynamic control law(11). Bottom graph: Time histories of the states $x_{2}(t)$ and $x_{3}(t)$ in the original $x$-coordinates in dashed-dotted grey and solid dark lines, respectively.
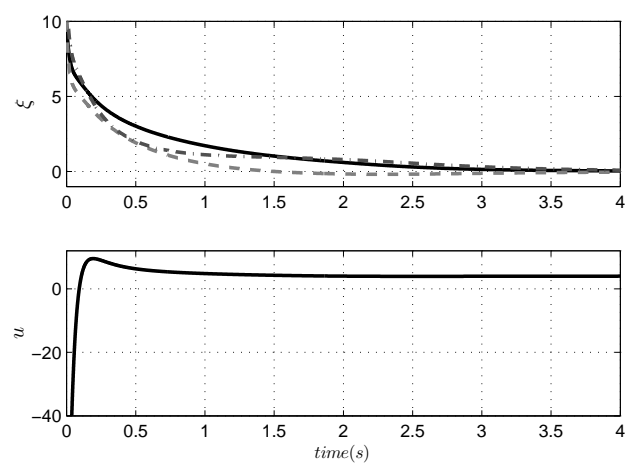

Figure 5. Top graph: Time histories of the dynamic extension $\xi_{1}(t)$ (solid), $\xi_{2}(t)$ (dash-dotted), $\xi_{3}(t)$ (dashed). Bottom graph: Time history of the control input $u$ in (19).

control law (19). The top graph of Figure 5 depicts the time histories of the states of the dynamic extension $\xi$ and the bottom graph the control law $u$ which is positive as required by the physics of the system.

\section{CONCLUSIONS}

The IDA-PBC design methodology for PCH systems has been revisited. An alternative solution to the problem has been proposed by exploiting the notion of algebraic solution of the matching equation. Such solution is instrumental for the construction of an auxiliary energy function defined in an extended state-space, without involving the solution of any partial differential equation. The extended energy function has been employed to pursue two different approaches. To begin with, under mild assumptions, we have provided a control law that (asymtptotically) stabilizes a desired equilibrium of the closed-loop system, without however preserving the $\mathrm{PCH}$ structure. Differently from the classical solution, the stabilizing control law is given in terms of a dynamic state-feedback. Then, under additional technical assumptions, the auxiliary energy function has been instrumental for the construction of a dynamic control law that retains the $\mathrm{PCH}$ structure of the extended system. As a noteworthy consequence of the latter result we have shown that if the matching equation does not admit a (classical) solution for a desired interconnection and damping structure, the auxiliary energy function may be exploited to construct a (classical) solution to matching equations corresponding to an interconnection and damping structure which can be written as the sum of the desired matrices and continuous perturbation terms vanishing at the equilibrium. Finally, it is shown that, under the standard assumptions, the proposed approach yields the classical solution in the case of linear PCH systems. The approach has been validated on a magnetic levitated system and a third order food-chain system by assigning an interconnection and damping structure that cannot be imposed with the standard approach. Future research directions include the application of the proposed approach to classes of port-controlled Hamiltonian systems, such as underactuated mechanical systems.

\section{REFERENCES}

[1] J. A. Acosta and A. Astolfi. On the PDEs arising in IDA-PBC. In Proc. of the 48th IEEE Conf. Decision and Control, Shanghai, China, pages $2132-2137,2009$.

[2] J. A. Acosta, R. Ortega, A. Astolfi, and A. D. Mahindrakar. Interconnection and damping assignment passivity-based control of mechanical systems with underactuation degree one. Automatic Control, IEEE Transactions on, 50(12):1936-1955, 2005.

[3] K. M. Anstreicher and M. H. Wright. A note on the augmented Hessian when the reduced Hessian is semidefinite. SIAM Journal on Optimization, 11:243-253, 2000.

[4] A. Astolfi and R. Ortega. Dynamic extension is unnecessary for stabilization via interconnection and damping assignment passivitybased control. Systems and Control Letters, 58:133-135, 2009.

[5] D. Auckly, L. Kapitanski, and W. White. Control of nonlinear underactuated systems. arXiv preprint math/9901140, 1999.

[6] G. Blankenstein, R. Ortega, and A. van ver Schaft. The matching conditions of controlled Lagrangians and IDA-passivity based control. International Journal of Control, 75(9):645-665, 2002.

[7] A. M. Bloch, D. E. Chang, N. E. Leonard, and J. E. Marsden. Controlled Lagrangians and the stabilization of mechanical systems. II. Potential shaping. Automatic Control, IEEE Transactions on, 46(10):1556-1571, 2001. 
[8] A. M. Bloch, N. E Leonard, and J. E Marsden. Controlled Lagrangians and the stabilization of mechanical systems. I. The first matching theorem. Automatic Control, IEEE Transactions on, 45(12):2253-2270, 2000.

[9] D. E. Chang, A. M Bloch, N. E. Leonard, J. E. Marsden, and C. A. Woolsey. The equivalence of controlled Lagrangian and controlled Hamiltonian systems. ESAIM: Control, Optimisation and Calculus of Variations, 8(Specia):393-422, 2002.

[10] K. Fujimoto, K. Sakurama, and T. Sugie. Trajectory tracking control of port-controlled Hamiltonian systems via generalized canonical transformations. Automatica, 39(12):2059-2069, 2003.

[11] M. Galaz, R. Ortega, A. S. Bazanella, and A. M. Stankovic. An energyshaping approach to the design of excitation control of synchronous generators. Automatica, 39(1):111-119, 2003.

[12] J. Hamberg. General matching conditions in the theory of controlled Lagrangians. In Decision and Control, 1999. Proceedings of the 38th IEEE Conference on, volume 3, pages 2519-2523. IEEE, 1999.

[13] J. Hofbauer and K. Sigmund. Evolutionary games and population dynamics. Cambridge University Press, 1998.

[14] D. Karagiannis, M. Sassano, and A. Astolfi. Dynamic scaling and observer design with application to adaptive control. Automatica, 45(12):2883-2889, 2009.

[15] P. Kotyczka. Local linear dynamics assignment in IDA-PBC. Automatica, 49(4):1037-1044, 2013.

[16] B. Maschke, R. Ortega, and A. van der Schaft. Energy-based Lyapunov functions for forced Hamiltonian systems with dissipation. IEEE Transactions on Automatic Control, 45(8):1498-1502, 2000.

[17] K. Nunna, M. Sassano, and A. Astolfi. Constructive interconnection and damping assignment for port-controlled Hamiltonian. In Proc. of the American Control Conference, pages 1810-1815, 2013.

[18] R. Ortega. Passivity-based control of Euler-Lagrange systems: mechanical, electrical and electromechanical applications. Springer, 1998.

[19] R. Ortega, A. Astolfi, G. Bastin, and H. Rodriguez. Output-feedback regulation of mass-balance systems. New Directions in Nonlinear Observer Design, 1999.

[20] R. Ortega, A. Astolfi, G. Bastin, and H. Rodriguez. Stabilization of food-chain systems using a port-controlled hamiltonian description. In American Control Conference, 2000. Proceedings of the 2000, volume 4, pages 2245-2249 vol.4, 2000.

[21] R. Ortega, A. Van der Schaft, I. Mareels, and B. Maschke. Putting energy back in control. IEEE Control Systems Magazine, 21(2):18-33, 2001.

[22] R. Ortega and M.W. Spong. Stabilization of underactuated mechanical systems via Interconnection and Damping Assignment. IEEE Transactions on Automatic Control, 47:1218-1233, 2000.

[23] R. Ortega, A. van der Schaft, F. Castanos, and A. Astolfi. Control by interconnection and standard passivity-based control of port-Hamiltonian systems. Automatic Control, IEEE Transactions on, 53(11):2527-2542, 2008.

[24] R. Ortega, A. van der Schaft, B. Maschke, and G. Escobar. Interconnection and damping assignment passivity-based control of port-controlled Hamiltonian systems. Automatica, 38(4):585-596, 2002.

[25] R. Ortega, A. van der Schaft, and B. M Maschke. Stabilization of portcontrolled Hamiltonian systems via energy balancing. In Stability And Stabilization Of Nonlinear Systems, pages 239-260. Springer, 1999.

[26] S. Prajna, A. van der Schaft, and G. Meinsma. An LMI approach to stabilization of linear port-controlled Hamiltonian systems. Systems \& Control Letters, 45(5):371-385, 2002.

[27] H. Rodriguez, R. Ortega, and I. Mareels. Nonlinear control of magnetic levitation systems via energy balancing. In Proc. Amer. Control Conf, 2000.

[28] M. Sassano and A. Astolfi. Dynamic approximate solutions of the HJ inequality and the HJB equation for input-affine nonlinear systems. IEEE Transactions on Automatic Control, 57(10):2490-2503, 2012.

[29] M. Sassano and A. Astolfi. Approximate finite horizon optimal control without PDE's. Systems and Control Letters, 62(2):97-103, 2013.

[30] M. Sassano and A. Astolfi. Dynamic lyapunov functions. Automatica, 49(4):1058-1067, 2013.

[31] M. Sassano and A. Astolfi. Dynamic generalized controllability and observability functions with applications to model reduction and sensor deployment. Automatica, 50(5):1349-1359, 2014.

[32] M. Takegaki and S. Arimoto. A new feedback method for dynamic control of manipulators. Journal of Dynamic Systems, Measurement, and Control, 103:119, 1981.

[33] A. van der Schaft. $L_{2}$-Gain and Passivity Techniques in Nonlinear Control. Springer-Verlag, London, 2nd edition, 2000.

[34] A. van der Schaft. Port-controlled Hamiltonian systems: towards a theory for control and design of nonlinear physical systems. Journal of the Society of Instrument and Control Engineers of Japan (SICE), 39(2):91-98, 2000.
[35] G. Viola, R. Ortega, R. Banavar, J. A. Acosta, and A. Astolfi. Total energy shaping control of mechanical systems: simplifying the matching equations via coordinate changes. Automatic Control, IEEE Transactions on, 52(6):1093-1099, 2007.

[36] C. Woolsey, C. K. Reddy, A. M. Bloch, D. E. Chang, N. E. Leonard, and J. E. Marsden. Controlled Lagrangian systems with gyroscopic forcing and dissipation. European Journal of Control, 10(5):478-496, 2004.

[37] C. A. Woolsey and N. E. Leonard. Stabilizing underwater vehicle motion using internal rotors. Automatica, 38(12):2053 - 2062, 2002.

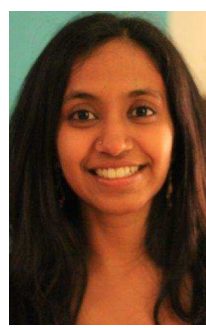

Kameswarie Nunna received the B.Eng degree in Instrumentation and Control Engineering from National Institute of Technology, Trichy, India in 2009. She obtained the M.Sc. degree with distinction in Control Systems from Imperial College London in 2010, and the Ph.D degree in Constructive IDAPBC with applications from the Control and Power group of the Department of Electrical and Electronic Engineering under the guidance of Prof. Alessandro Astolfi. She is currently working at The MathWorks as an Application Support Engineer. Her research interests include nonlinear control, Hamiltonian systems, nonlinear observer design, and modelling of electromechanical systems.

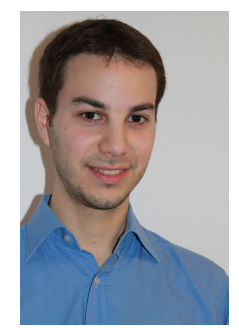

Mario Sassano was born in Rome, Italy, in 1985. He received the B.S degree in Automation Systems Engineering and the M.S degree in Systems and Control Engineering from the University of Rome "La Sapienza", Italy, in 2006 and 2008, respectively. In 2012 he obtained a Ph.D. degree by Imperial College London, UK, where he had been a research assistant at the Department of Electrical and Electronic Engineering since 2009. Currently he is a postdoctoral research assistant at the University of Rome "Tor Vergata", Italy. His research interests are focused on nonlinear observer design, optimal control theory with applications to mechatronical systems and image processing.

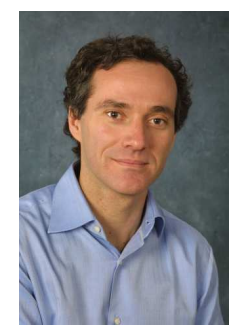

Alessandro Astolfi was born in Rome, Italy, in 1967. He graduated in electrical engineering from the University of Rome in 1991. In 1992 he joined ETH-Zurich where he obtained a M.Sc in Information Theory in 1995 and the Ph.D. degree with Medal of Honour in 1995 with a thesis on discontinuous stabilization of nonholonomic systems. In 1996 he was awarded a Ph.D. from the University of Rome "La Sapienza" for his work on nonlinear robust control. Since 1996 he is with the Electrical and Electronic Engineering Department of Imperial College, London (UK), where he is currently Professor in Non-linear Control Theory. From 1998 to 2003 he was also an Associate Professor at the Dept. of Electronics and Information of the Politecnico of Milano. Since 2005 he is also Professor at Dipartimento di Informatica, Sistemi e Produzione, University of Rome Tor Vergata. He has been visiting lecturer in "Nonlinear Control" in several universities, including ETH-Zurich (1995-1996); Terza University of Rome (1996); Rice University, Houston (1999); Kepler University, Linz (2000); SUPELEC, Paris (2001). His research interests are focused on mathematical control theory and control applications, with special emphasis for the problems of discontinuous stabilization, robust stabilization, robust control and adaptive control. He is author of more than 70 journal papers, of 20 book chapters and of over 160 papers in refereed conference proceedings. $\mathrm{He}$ is author (with D. Karagiannis and R. Ortega) of the monograph Nonlinear and Adaptive Control with Applications (Springer Verlag). He is Associate Editor of Systems and Control Letters, Automatica, IEEE Trans. Automatic Control, the International Journal of Control, the European Journal of Control, the Journal of the Franklin Institute, and the International Journal of Adaptive Control and Signal Processing. He has also served in the IPC of various international conferences. 